\title{
Transient global amnesia after radiofrequency catheter ablation of supraventricular tachycardia: a case report
}

\author{
Shuai Sun, Qianwen Huang, Xiongbiao Chen, Qianqian Liu, Chun Wang \\ Department of Cardiology, Peking University Shenzhen Hospital, Shenzhen, China \\ Correspondence to: Chun Wang. Department of Cardiology, Peking University Shenzhen Hospital, Shenzhen 518036, China. \\ Email: wangchun_6646@163.com.
}

\begin{abstract}
Transient global amnesia (TGA) is a neurological disorder characterized by sudden onset of anterograde amnesia with or without retrograde amnesia, lasting less than 24 hours, without other clinical neurological dysfunction. TGA with short duration and benign prognosis is usually neglected in clinical practice. Transient total amnesia after radiofrequency ablation is rare and its etiology is unknown. We report a case of 27-year-old man who experienced TGA after radiofrequency catheter ablation of supraventricular tachycardia. The patient had no other cognitive and motor impairment except for memory impairment. The symptom lasted for about six hours and relieved without recurrence. Nervous system examination showed that 12 pairs of cranial nerves were normal, the muscle strength and muscle tone of the limbs were normal, physiological reflexes existed, and no pathological reflexes were elicited. Tests were performed immediately and normally including blood routine examination, liver and kidney function, electrolyte, blood glucose, thyroid function, blood coagulation function, D-dimer, myocardial injury markers, blood gas analysis and other hematological. There is no abnormality in electrocardiogram (ECG), chest X-ray, cervical vascular ultrasound, and cardiac color Doppler ultrasound examination. Head magnetic resonance examination magnetic resonance imaging (MRI) showed dots in right frontal lobe and bilateral ventricles in T2-weighted images. There was no cerebral infarction and cerebral hemorrhage. The patient received low flow oxygen inhalation and aspirin $300 \mathrm{mg}$ orally. The outcome of patient with TGA is benign. There are still many unsolved mysteries worthy of long-term follow-up.
\end{abstract}

Keywords: Transient global amnesia (TGA); paroxysmal supraventricular tachycardia; radiofrequency catheter ablation

Submitted Nov 03, 2020. Accepted for publication Feb 21, 2021.

doi: $10.21037 / \mathrm{cdt}-20-895$

View this article at: http://dx.doi.org/10.21037/cdt-20-895

\section{Introduction}

Transient global amnesia (TGA) is a neurological disorder characterized by sudden onset of anterograde amnesia with or without retrograde amnesia, lasting less than 24 hours, without other clinical neurological dysfunction. TGA with short duration and benign prognosis is usually neglected in clinical practice. Transient total amnesia after radiofrequency ablation is rare and its etiology is unknown. We report a case of 27-year-old man who experienced TGA after radiofrequency catheter ablation of supraventricular tachycardia. The patient had no other cognitive and motor impairment except for memory impairment. The symptom lasted for about six hours and relieved without recurrence.

We present the study in accordance with the CARE reporting checklist (available at http://dx.doi.org/10.21037/ cdt-20-895).

\section{Case presentation}

A 27-year-old male patient was admitted to the hospital due to "onset of palpitations for half a year with aggravation for 1 day" on September 23, 2020. There are nine episodes of paroxysmal palpitations with 10 minutes to 2 hours each 


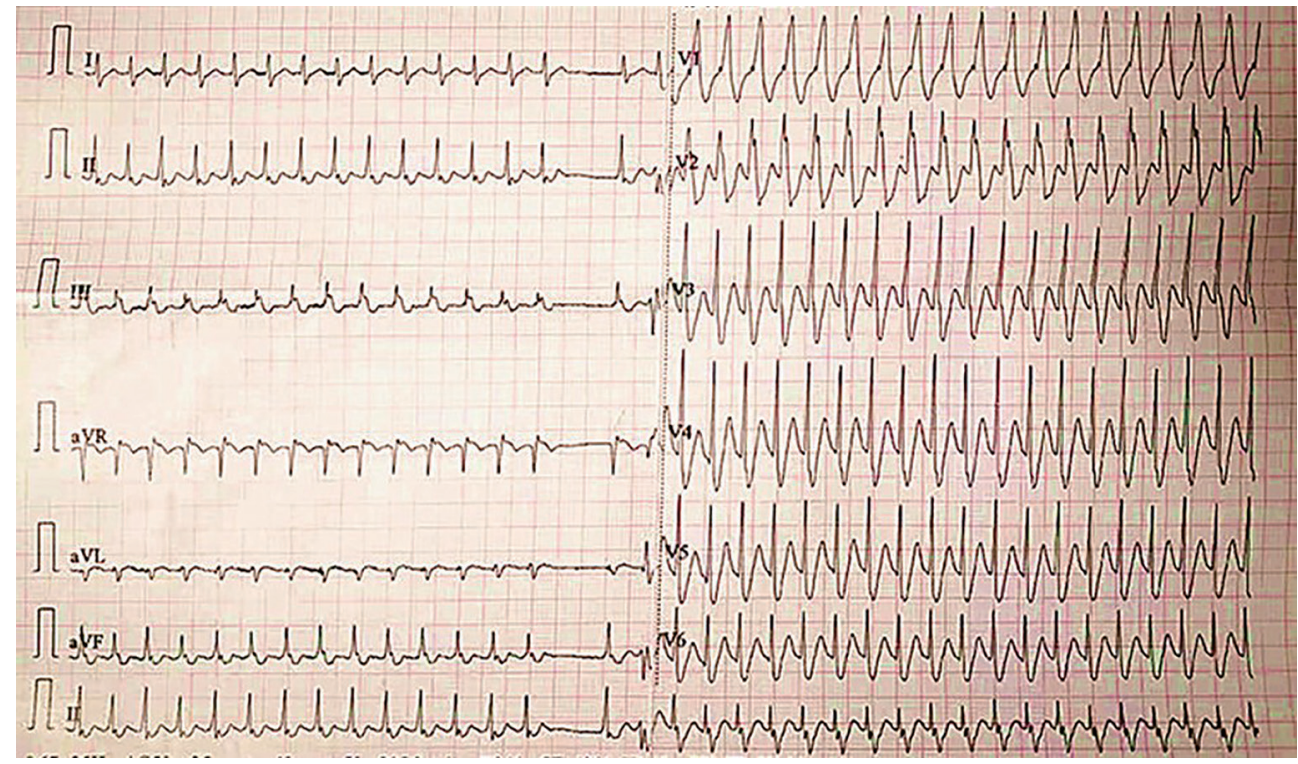

Figure 1 12-lead electrocardiogram of paroxysmal supraventricular tachycardia.

time and abrupt termination without syncope in the past 6 months. Electrocardiogram (ECG) indicates tachycardia with narrow QRS, ventricular rate $216 \mathrm{bpm}$ at the onset on August 26, 2020 (Figure 1). ECG diagnosis is paroxysmal supraventricular tachycardia. He does not have history of smoking, alcohol abuse, drug abuse and drug allergy.

\section{Operation process}

Routine preoperative examination showed no abnormality after admission. Intracardiac electrophysiological examination and radiofrequency ablation were performed on September 24, 2020. The procedure includes routine disinfection, cloth covering, local anesthesia with $1 \%$ lidocaine, puncturing right internal jugular vein and right femoral vein, and sending 10-pole and 4-pole mapping electrodes to coronary sinus and right ventricular apex respectively through $6 \mathrm{~F}$ sheath tube.

Spontaneous tachycardia with narrow QRS occurred during the operation, and the retrograde $\mathrm{A}$ wave in the cavity was shown in lead of CS3 and 4 excitement (Figure 2). Ventricular and atrial stimulation showed eccentric nondecreasing conduction, which can induce the above narrow QRS tachycardia, and the retrograde A wave in lead of CS3 and 4 activation. It was proved to be the atrioventricular reentry tachycardia mediated by the occult accessory pathway of the left free wall. The atrial septum was punctured and then intravenous heparin 3,000 $\mathrm{U}$ was injected. The ablation electrode catheter was sent to the left ventricular sub-mitral free wall CS3 and 4 through the atrial septal route (Figure 3). Ventriculoatrial (V-A) fusion site is the target in ventricular pacing. Thirty to fifty $\mathrm{W}, 55^{\circ} \mathrm{C}$ temperature (T)-controlled discharge, ventricular atrial separation showed within 2 seconds of right ventricular pacing, and the discharge was consolidated at the target point for 180 seconds. Repeat intracardiac electrophysiological examination, ventricle S1S1 400 milliseconds showed V-A block, and neither atrial nor ventricular program stimulation could induce tachycardia. The sheath was removed, local compression was performed to stop bleeding after the operation. The operation lasted for 30 minutes. The patient was sent back to the ward without discomfort.

However, his family members found that the patient repeatedly asking the same questions every 5 minutes after returning to the ward, such as "Where am I?", "What happened?". The patient had time and location disorientation. He was not sure when and where he was. He could not recall the admission and surgery procedure. He did not have headache, dizziness, palpitations, nausea, vomiting, hemianopia, aphasia, loss of consciousness, nor other symptoms such as limited physical activity. Physical examination: T, $36.5^{\circ} \mathrm{C}$; P, $70 \mathrm{bpm}$; RR, $18 \mathrm{bpm}$; blood pressure (BP), 120/70 $\mathrm{mmHg}$. The patient had clear speech, symmetrical facial pattern, independent posture, no dry and wet rales in both lungs, and the heart rhythm was normal. There was no pathological murmur in the auscultation 


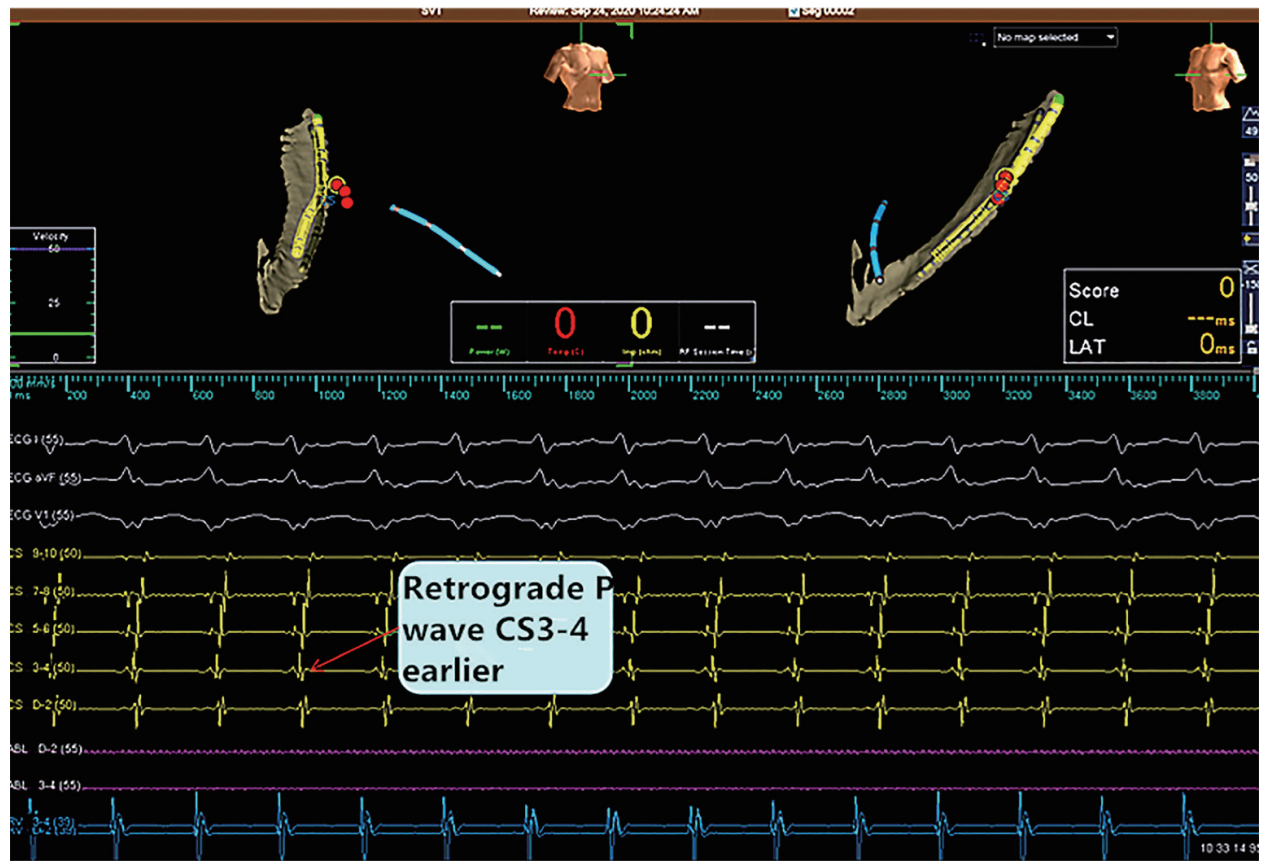

Figure 2 Mapping electrogram during radiofrequency ablation.

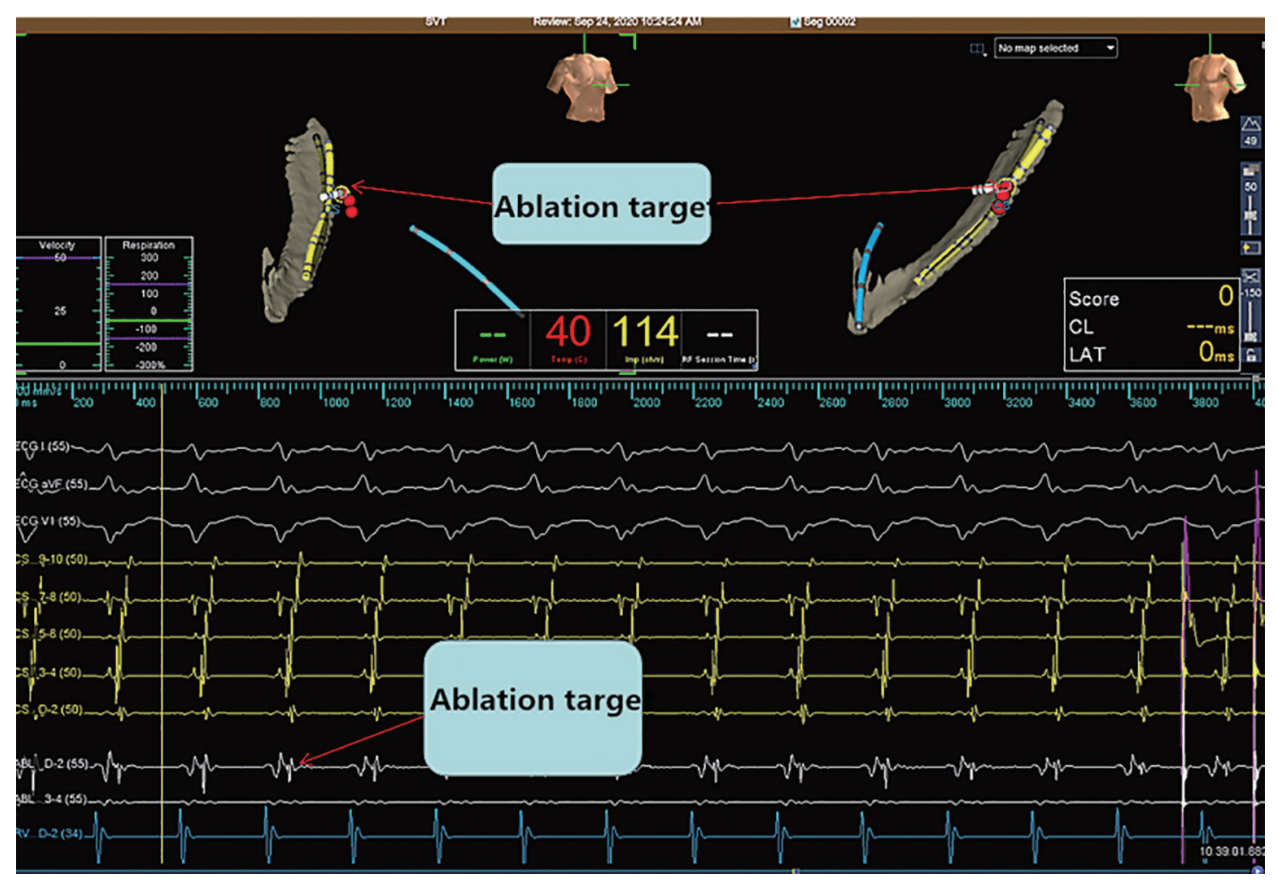

Figure 3 Mapping electrogram during radiofrequency ablation.

area of each valve. The abdominal examination showed no abnormality. The wound dressing at the puncture site of right femoral vein was dry without blood. The pulse of dorsalis pedis artery of both lower limbs was normal. Nervous system examination shows that 12 pairs of cranial nerves were normal, the muscle strength and muscle tone 


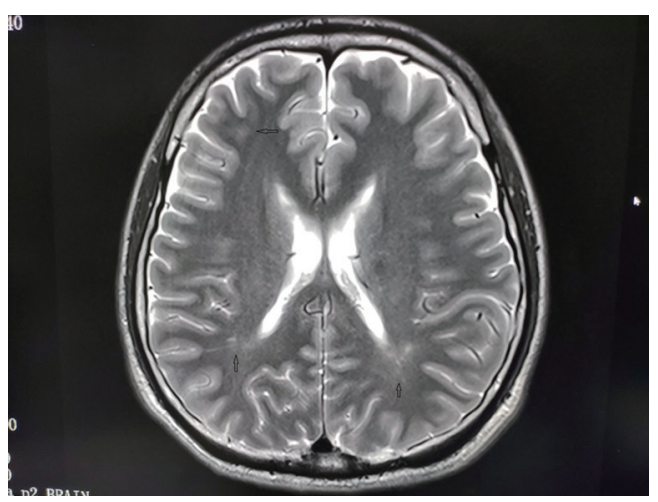

Figure 4 Head MRI: the arrow indicated dot-shaped hyperintensity shadows in T2-weighted images. MRI, magnetic resonance imaging.

of the limbs were normal, physiological reflexes existed, no pathological reflexes were elicited.

Tests were performed immediately and normal including blood routine examination, liver and kidney function, electrolyte, blood glucose, thyroid function, blood coagulation function, D-dimer, myocardial injury markers, blood gas analysis and other hematological. There is no abnormality in ECG, chest $\mathrm{X}$-ray, cervical vascular ultrasound, and cardiac color Doppler ultrasound examination. Head magnetic resonance examination MRI shows (Figure 4) dots in right frontal lobe and bilateral ventricles in $\mathrm{T} 2$-weighted images. There was no cerebral infarction and cerebral hemorrhage. The patient received low flow oxygen inhalation and aspirin $300 \mathrm{mg}$ orally. The symptom lasted for about six hours and relieved. The patient was discharged two days after the operation, and was followed up one week later. He did not have the above symptom again.

All procedures performed in studies involving human participants were in accordance with the ethical standards of the institutional and/or national research committee(s) and with the Helsinki Declaration (as revised in 2013). Written informed consent was obtained from the patient.

\section{Discussion}

Amnesia is a form of memory disorder, which mainly shows anterograde amnesia and retrograde amnesia with normal intelligence. Anterograde amnesia refers to the poor memory of recent events and the inability to retain the newly acquired information, that is, the lack of the ability to transform short-term memory into long-term memory. Retrograde amnesia refers to forget past events, and new memories can be formed. TGA refers to sudden anterograde amnesia with or without retrograde amnesia, lasting less than 24 hours, without other clinical neurological dysfunction $(1,2)$. The current diagnosis of TGA mainly refers to the standard established by Hodges et al. (3), including confirmed by witnesses; anterograde memory loss; clear consciousness; no other cognitive impairments except for memory impairment; complete insight; no positive signs on neurological physical examination, and no recent history of brain trauma and seizures. Memory impairment recovers within 24 hours without other causes. It is related to brain hippocampus dysfunction. The hippocampus of the brain is an important organ for memory formation and preservation (4). It is mainly responsible for transforming short-term memory into long-term memory, which is distributed to different parts of the brain for memory storage. In addition, the hippocampus is responsible for orientation. From an anatomical point of view, the hippocampus spans between the left and right brains, locates between the cerebral thalamus and the medial temporal lobe. It is often seen as a medial bulge in the temporal horn of the lateral ventricle, which is susceptible to hypoxia.

After radiofrequency ablation of paroxysmal supraventricular tachycardia, the patient presented with disorientation, anterograde amnesia and retrograde amnesia. Anterograde amnesia is characterized by poor memory of recent events, inability to retain the newly acquired information and repeated asking the same questions. Retrograde amnesia is characterized by retrospective memory impairment and forgetting the process of admission and operation. The vital signs of the patient were stable after the onset of the disease, and no abnormality was found in the physical examination, hematology test, ECG, chest X-ray, neck vascular ultrasound, and cardiac color Doppler ultrasound. Head MRI examination shows dot hyperintensity shadows in T2-weighted images of the right frontal lobe and bilateral ventricles. No new cerebral infarction and cerebral hemorrhage were found.

According to the above symptoms and examination results, the diagnosis was consistent with TGA (3). Chou et al. report a similar case of paroxysmal supraventricular tachycardia who underwent TGA at the end of ablation. Characteristic MRI findings include reversible small punctate diffusion-weighted imaging (DWI) lesions over the temporal lobe, thalamus, or cerebellum and especially the hippocampus (5). Cui et al. (6) retrospectively analyzed the predisposing factors of 42 cases of TGA patients, among which 16 cases were induced by Valsalva action, which 
may be related to vagus nerve excitement, disturbance of electrical signals in the brain, and destruction of amino acid, protein synthesis and metabolism. Combining the diagnosis and treatment process of this patient, the possible factors for TGA were analyzed: (I) insufficient anticoagulation during the operation, microthromboembolism; (II) rapid ventricular rate (220 beats/min) during paroxysmal supraventricular tachycardia which causes transient cerebral ischemia. The above analysis is only speculation and there is not enough evidence to prove it.

TGA was first reported by French scientist Courjon et al. (7) in 1956. So far, the etiology and mechanism of the disease are still unclear $(8,9)$. If the TGA attack is related to the Valsalva maneuver, why the patient does not have symptoms after repeating the maneuver? If it is related to microthrombosis, why all the patients previously reported recover within 24 hours? If it is related to the frequency of tachycardia, why the TGA episode did not happen in every tachycardia attack? If it is related to surgery, the surgical process is a routine operation. During the operation, the patient has recurrent atrioventricular reentrant tachycardia. High-frequency, high-energy and strong stimulation are not performed. The ablation target is found quickly, and the ablation is successful. The patient has no nausea during the operation. According to the definition, TGA is a selflimiting state that can be relieved without intervention. Therefore, there is no specific treatment $(10,11)$. Whether drug therapy can shorten the duration of symptoms is inconclusive. We have treatment according to the possible cause, such as: low-flow oxygen inhalation, aspirin antiplatelet aggregation. The patient's symptoms are relieved after 6 hours when time and space positioning functions are restored, and new information storage function is preserved.

\section{Conclusions}

TGA does not require specific treatment. The general longterm outcome of patients with TGA appears to be benign. There are still many unsolved mysteries worthy of longterm follow-up.

\section{Acknowledgments}

Funding: None.

\section{Footnote}

Reporting Checklist: The authors have completed the CARE reporting checklist. Available at http://dx.doi.org/10.21037/ cdt-20-895

Conflicts of Interest: All authors have completed the ICMJE uniform disclosure form (available at http://dx.doi. org/10.21037/cdt-20-895). The authors have no conflicts of interest to declare.

Etbical Statement: The authors are accountable for all aspects of the work in ensuring that questions related to the accuracy or integrity of any part of the work are appropriately investigated and resolved. All procedures performed in studies involving human participants were in accordance with the ethical standards of the institutional and/or national research committee(s) and with the Helsinki Declaration (as revised in 2013). Written informed consent was obtained from the patient.

Open Access Statement: This is an Open Access article distributed in accordance with the Creative Commons Attribution-NonCommercial-NoDerivs 4.0 International License (CC BY-NC-ND 4.0), which permits the noncommercial replication and distribution of the article with the strict proviso that no changes or edits are made and the original work is properly cited (including links to both the formal publication through the relevant DOI and the license). See: https://creativecommons.org/licenses/by-nc-nd/4.0/.

\section{References}

1. Meyer IA, Wintermark M, Démonet JF, et al. CTP in Transient Global Amnesia: A Single-Center Experience of 30 Patients. AJNR Am J Neuroradiol 2015;36:1830-3.

2. Graff-Radford J, Clapp AJ, Lanzino G, et al. Transient amnesia after coiling of a posterior circulation aneurysm. Neurocrit Care 2013;18:245-7.

3. Hodges JR, Warlow CP. Syndromes of transient amnesia: towards a classification. A study of 153 cases. J Neurol Neurosurg Psychiatry 1990;53:834-43.

4. Bartsch T, Deuschl G. Transient global amnesia: functional anatomy and clinical implications. Lancet Neurol 2010;9:205-14.

5. Chou CY, Chen CP, Huang CH. Transient global amnesia after ablation of the left lateral accessory pathway. Indian Pacing Electrophysiol J 2012;12:69-72.

6. Cui W, Liu L, Jia S, et al. Clinical and imaging character of transient global amnesia. Journal of China-Japan Friendship Hospital 2019;33:343-5. 
7. Courjon J, Guyotat J. Amnesic strokes. J Med Lyon 1956;37:697-701.

8. Tiu C, Terecoasă EO, Grecu N, et al. Transient Global Amnesia After Cerebral Angiography With Iomeprol: A Case Report. Medicine (Baltimore) 2016;95:e3590.

9. Lee BH. Transient global amnesia following carotid artery

Cite this article as: Sun S, Huang Q, Chen X, Liu Q, Wang C. Transient global amnesia after radiofrequency catheter ablation of supraventricular tachycardia: a case report. Cardiovasc Diagn Ther 2021;11(2):472-477. doi: 10.21037/cdt-20-895 stenting: A case report. Radiol Case Rep 2020;15:1159-63.

10. Brown J. ED evaluation of transient global amnesia. Ann Emerg Med 1997;30:522-6.

11. Spiegel DR, Smith J, Wade RR, et al. Transient global amnesia: current perspectives. Neuropsychiatr Dis Treat 2017;13:2691-703. 